\title{
Gauge invariance of sedeonic equations of massive and massless fields
}

\author{
Victor L. Mironov* and Sergey V. Mironov \\ Institute for physics of microstructures RAS, Nizhniy Novgorod, Russia
}

(Submitted on July 3, revised on 6 July 2015)

\begin{abstract}
We discuss the gradient gauge invariance for the massive and massless fields described by sedeonic second-order and first-order wave equations.
\end{abstract}

\section{Introduction}

The generalization of scalar Klein-Gordon wave equation and spinor Dirac equation on the basis of wider class of multicomponent wave functions is the subject of intensive investigations. In particular, in recent years many attempts have been made to generalize these equations using different algebras of hypercomplex numbers, such as four-component quaternions (including scalar and vector) [1-4] and eight-component octonions (including scalar, vector (polar vector), pseudoscalar and pseudovector (axial vector)) [5-7]. The authors discuss the possibility of constructing the field equations similar to the equations of electrodynamics but with a massive "photon". However, the resulting Proca-Maxwell equations are not gauge invariant [8-10]. On the other hand, a consistent relativistic approach implies equally the space and time symmetries that require the consideration of the extended sixteen-component space-time algebras. There are a few approaches in the development of field theory on the basis of sixteen-component structures. One of them is the application of hypernumbers sedenions, which are obtained from octonions by Cayley-Dickson extension procedure [11-15]. But the essential imperfection of sedenions is their nonassociativity. Another approach is based on the application of hypercomplex multivectors generating associative space-time Clifford algebras [16]. However, the application of such sixteen-component structures is considered in general as the abstract algebraic scheme enabling the factorization of Klein-Gordon and Dirac operators.

Recently we proposed the alternative approach based on sixteen-component sedeons generating noncommutative but associative scalar-vector algebra [17]. The sedeons take into account the properties of physical values with respect to the space-time inversion and realize the scalar-vector representation of Poincare group. In present paper, we apply the sedeonic approach for the construction of symmetric scalar-vector equations of massive and massless fields described by sedeonic second-order and first-order wave equations. The main attention is focused on the discussion of gradient gauge invariance of field equations.

\section{Sedeonic space-time algebra}

To begin with we briefly review the basic properties of sedeons. The sedeonic algebra encloses four groups of values, which are differed with respect to spatial and time inversion.

\footnotetext{
*E-mail: mironov@ipmras.ru
} 
1. Absolute scalars $(A)$ and absolute vectors $(\vec{A})$ are not transformed under spatial and time inversion.

2. Time scalars $\left(B_{\mathbf{t}}\right)$ and time vectors $\left(\vec{B}_{\mathbf{t}}\right)$ are changed (in sign) under time inversion and are not transformed under spatial inversion.

3. Space scalars $\left(C_{\mathbf{r}}\right)$ and space vectors $\left(\vec{C}_{\mathbf{r}}\right)$ are changed under spatial inversion and are not transformed under time inversion.

4. Space-time scalars $\left(D_{\mathbf{t r}}\right)$ and space-time vectors $\left(\vec{D}_{\mathbf{t r}}\right)$ are changed under spatial and time inversion.

The indexes $\mathbf{t}$ and $\mathbf{r}$ indicate the transformations ( $\mathbf{t}$ for time inversion and $\mathbf{r}$ for spatial inversion), which change the corresponding values. The space-time sedeon $\tilde{\mathbf{S}}$ is defined by the following expression:

$$
\tilde{\mathbf{S}}=A+\vec{A}+B_{\mathbf{t}}+\vec{B}_{\mathbf{t}}+C_{\mathbf{r}}+\vec{C}_{\mathbf{r}}+D_{\mathbf{t r}}+\vec{D}_{\mathbf{t r}} .
$$

Here and further we indicate the sedeon by bold symbol with wave. The components of sedeon (1) can be written in the sedeonic space-time basis as

$$
\begin{aligned}
& A=\mathbf{e}_{\mathbf{0}} A \mathbf{a}_{\mathbf{0}}, \\
& \vec{A}=\mathbf{e}_{\mathbf{0}}\left(A_{1} \mathbf{a}_{\mathbf{1}}+A_{2} \mathbf{a}_{\mathbf{2}}+A_{3} \mathbf{a}_{\mathbf{3}}\right), \\
& B_{\mathbf{t}}=\mathbf{e}_{\mathbf{t}} B \mathbf{a}_{\mathbf{0}}, \\
& \vec{B}_{\mathbf{t}}=\mathbf{e}_{\mathbf{t}}\left(B_{1} \mathbf{a}_{\mathbf{1}}+B_{2} \mathbf{a}_{\mathbf{2}}+B_{3} \mathbf{a}_{\mathbf{3}}\right), \\
& C_{\mathbf{r}}=\mathbf{e}_{\mathbf{r}} C \mathbf{a}_{\mathbf{0}}, \\
& \vec{C}_{\mathbf{r}}=\mathbf{e}_{\mathbf{r}}\left(C_{1} \mathbf{a}_{\mathbf{1}}+C_{2} \mathbf{a}_{\mathbf{2}}+C_{3} \mathbf{a}_{\mathbf{3}}\right), \\
& D_{\mathbf{t r}}=\mathbf{e}_{\mathbf{t r}} D \mathbf{a}_{\mathbf{0}}, \\
& \vec{D}_{\mathbf{t r}}=\mathbf{e}_{\mathbf{t r}}\left(D_{1} \mathbf{a}_{\mathbf{1}}+D_{2} \mathbf{a}_{\mathbf{2}}+D_{3} \mathbf{a}_{\mathbf{3}}\right),
\end{aligned}
$$

where values $\mathbf{a}_{\mathbf{0}}, \mathbf{a}_{\mathbf{1}}, \mathbf{a}_{\mathbf{2}}, \mathbf{a}_{\mathbf{3}}$ are scalar-vector basis $\left(\mathbf{a}_{\mathbf{0}} \equiv 1\right.$ is absolute scalar unit and the values $\mathbf{a}_{\mathbf{1}}, \mathbf{a}_{\mathbf{2}}, \mathbf{a}_{\mathbf{3}}$ are absolute unit vectors generating the right Cartesian basis) and values $\mathbf{e}_{\mathbf{0}}, \mathbf{e}_{\mathbf{t}}, \mathbf{e}_{\mathbf{r}}, \mathbf{e}_{\mathbf{t r}}$ are space-time basis $\left(\mathbf{e}_{\mathbf{0}} \equiv 1\right.$ is a absolute scalar unit; $\mathbf{e}_{\mathbf{t}}$ is a time unit; $\mathbf{e}_{\mathbf{r}}$ is a space unit; $\mathbf{e}_{\mathbf{t r}}$ is a space-time unit). Further we will omit the units $\mathbf{a}_{\mathbf{0}}$ and $\mathbf{e}_{\mathbf{0}}$ for simplicity.

The multiplication and commutation rules for the sedeonic absolute unit vectors $\mathbf{a}_{\mathbf{1}}, \mathbf{a}_{\mathbf{2}}, \mathbf{a}_{\mathbf{3}}$ and space-time units $\mathbf{e}_{\mathbf{t}}, \mathbf{e}_{\mathbf{r}}, \mathbf{e}_{\mathbf{t r}}$ are presented in the tables 1 and 2 respectively (in the tables and further the value $i$ is the imaginary unit $\left(i^{2}=-1\right)$ ). Note that sedeonic units $\mathbf{e}_{\mathbf{t}}, \mathbf{e}_{\mathbf{r}}, \mathbf{e}_{\mathbf{t r}}$ commute with $\mathbf{a}_{1}, \mathbf{a}_{2}, \mathbf{a}_{3}$.

Thus the sedeon $\tilde{\mathbf{S}}$ is the complicated space-time object consisting of absolute scalar, time scalar, space scalar, space-time scalar, absolute vector, time vector, space vector and space-time vector.

In sedeonic algebra we assume the Clifford multiplication of vectors. For example, the sedeonic product of two absolute vectors $\vec{A}$ and $\vec{B}$ can be presented in the following form:

$$
\vec{A} \vec{B}=(\vec{A} \cdot \vec{B})+[\vec{A} \times \vec{B}] .
$$

Here we denote the sedeonic scalar multiplication of two vectors (internal product) by symbol "." and round brackets

$$
(\vec{A} \cdot \vec{B})=A_{1} B_{1}+A_{2} B_{2}+A_{3} B_{3}
$$

and sedeonic vector multiplication (external product) by symbol " $\times$ " and square brackets

$$
[\vec{A} \times \vec{B}]=i\left(A_{2} B_{3}-A_{3} B_{2}\right) \mathbf{a}_{\mathbf{1}}+i\left(A_{3} B_{1}-A_{1} B_{3}\right) \mathbf{a}_{\mathbf{2}}+i\left(A_{1} B_{2}-A_{2} B_{1}\right) \mathbf{a}_{\mathbf{3}} .
$$

Note that in sedeonic algebra the definition of the vector product differs from analogous expression in Gibbs-Heaviside vector algebra. 
Table 1:

Table 1.

\begin{tabular}{cccc}
\hline & $\mathbf{a}_{1}$ & $\mathbf{a}_{2}$ & $\mathbf{a}_{3}$ \\
\hline $\mathbf{a}_{1}$ & 1 & $i \mathbf{a}_{3}$ & $-i \mathbf{a}_{2}$ \\
$\mathbf{a}_{2}$ & $-i \mathbf{a}_{3}$ & 1 & $i \mathbf{a}_{1}$ \\
$\mathbf{a}_{3}$ & $i \mathbf{a}_{2}$ & $-i \mathbf{a}_{1}$ & 1
\end{tabular}

Table 2:

Table 2.

\begin{tabular}{cccc}
\hline & $\mathbf{e}_{\mathbf{t}}$ & $\mathbf{e}_{\mathbf{r}}$ & $\mathbf{e}_{\mathbf{t r}}$ \\
\hline $\mathbf{e}_{\mathbf{t}}$ & 1 & $i \mathbf{e}_{\mathbf{t r}}$ & $-i \mathbf{e}_{\mathbf{r}}$ \\
$\mathbf{e}_{\mathbf{r}}$ & $-i \mathbf{e}_{\mathbf{t r}}$ & 1 & $i \mathbf{e}_{\mathbf{t}}$ \\
$\mathbf{e}_{\mathbf{t r}}$ & $i \mathbf{e}_{\mathbf{r}}$ & $-i \mathbf{e}_{\mathbf{t}}$ & 1 \\
\hline
\end{tabular}

\section{Sedeonic second-order equation for massive field}

Let us introduce the operators:

$$
\begin{aligned}
\partial & =\frac{1}{c} \frac{\partial}{\partial t} \\
\vec{\nabla} & =\frac{\partial}{\partial x} \mathbf{a}_{\mathbf{1}}+\frac{\partial}{\partial y} \mathbf{a}_{\mathbf{2}}+\frac{\partial}{\partial z} \mathbf{a}_{\mathbf{3}} \\
m & =\frac{m_{0} c}{\hbar}
\end{aligned}
$$

where $c$ is the speed of light, $m_{0}$ is the mass of quantum, $\hbar$ is the Planck constant. Then the sedeonic second-order wave equation for massive field [18] can be presented in the following symmetric form:

$$
\left(i \mathbf{e}_{\mathbf{t}} \partial-\mathbf{e}_{\mathbf{r}} \vec{\nabla}-i \mathbf{e}_{\mathbf{t r}} m\right)\left(i \mathbf{e}_{\mathbf{t}} \partial-\mathbf{e}_{\mathbf{r}} \vec{\nabla}-i \mathbf{e}_{\mathbf{t r}} m\right) \tilde{\mathbf{W}}=\tilde{\mathbf{J}},
$$

where $\tilde{\mathbf{W}}$ is a sedeonic field potential, $\tilde{\mathbf{J}}$ is a phenomenological sedeonic source of massive field. Denoting a new operator

$$
\widehat{\nabla}=\left(i \mathbf{e}_{\mathbf{t}} \partial-\mathbf{e}_{\mathbf{r}} \vec{\nabla}-i \mathbf{e}_{\mathbf{t r}} m\right),
$$

the wave equation (7) can be rewritten in very compact form :

$$
\widehat{\nabla} \widehat{\nabla} \tilde{\mathbf{W}}=\tilde{\mathbf{J}} .
$$

Additionally we can introduce the filed strengths

$$
\tilde{\mathbf{E}}=\widehat{\nabla} \tilde{\mathbf{W}},
$$

then the wave equation (7) takes the following form:

$$
\widehat{\nabla} \tilde{\mathbf{E}}=\tilde{\mathbf{J}} \text {. }
$$

The sedeonic equation (11) is the system of Maxwell-like equations for the massive filed strengths. Applying the operator $\widehat{\nabla}$ to $(11)$ we get the sedeonic wave equation for the field strengths

$$
\widehat{\nabla \nabla} \tilde{\mathbf{E}}=\widehat{\nabla} \tilde{\mathbf{J}}
$$


The equation (7) for potentials or the equations (11) (or (12)) for field strengths describe the massive field completely. From the viewpoint of symmetry these equations are invariant with respect to the following replacement of potential:

$$
\tilde{\mathbf{W}} \Rightarrow \tilde{\mathbf{W}}+\tilde{\mathbf{F}}+\widehat{\nabla} \tilde{\mathbf{G}}
$$

where $\tilde{\mathbf{F}}$ and $\tilde{\mathbf{G}}$ are arbitrary sedeons satisfy the following conditions:

$$
\begin{gathered}
\widehat{\nabla} \tilde{\mathbf{F}}=0, \\
\widehat{\nabla} \widehat{\nabla} \tilde{\mathbf{G}}=0 .
\end{gathered}
$$

The conditions (14) and (15) indicate that the potential $\tilde{\mathbf{W}}$ is defined up to an additive arbitrary function $\tilde{\mathbf{F}}$ satisfying the homogeneous first-order wave equation (sedeonic Dirac equation) and gradient of the arbitrary function $\tilde{\mathbf{G}}$, which satisfies the homogeneous second-order wave equation (sedeonic Klein-Gordon equation).

Besides, the equations (11) and (12) for field strengths are invariant with respect to the following substitution:

$$
\tilde{\mathbf{E}} \Rightarrow \tilde{\mathbf{E}}+\tilde{\mathbf{F}}+\widehat{\nabla} \tilde{\mathbf{G}} .
$$

In addition, increasing the order of the equation leads to the additional symmetry. Particularly, the equation (12) is invariant with respect to the replacement of source

$$
\tilde{\mathbf{J}} \Rightarrow \tilde{\mathbf{J}}+\tilde{\mathbf{F}}+\widehat{\nabla} \tilde{\mathbf{G}} \text {. }
$$

All the invariant relations (13), (16) and (17) are the consequences of specific properties of the operator $\widehat{\nabla}$.

Let us consider the equations for massive field in details. We choose the field potential as

$$
\tilde{\mathbf{W}}=i a_{1} \mathbf{e}_{\mathbf{t}}-i a_{2} \mathbf{e}_{\mathbf{r}}+a_{3}-i a_{4} \mathbf{e}_{\mathbf{t r}}+\vec{A}_{1} \mathbf{e}_{\mathbf{r}}+\vec{A}_{2} \mathbf{e}_{\mathbf{t}}-\vec{A}_{3} \mathbf{e}_{\mathbf{t r}}+i \vec{A}_{4},
$$

where components $a_{\mathrm{s}}$ and $\overrightarrow{A_{\mathrm{s}}}$ are real functions of coordinates and time. Here and further the index $\mathrm{s}=1,2,3,4$. Also we take the field source in the following form:

$$
\tilde{\mathbf{J}}=-i \rho_{1} \mathbf{e}_{\mathbf{t}}+i \rho_{2} \mathbf{e}_{\mathbf{r}}-\rho_{3}+i \rho_{4} \mathbf{e}_{\mathbf{t r}}-\vec{j}_{1} \mathbf{e}_{\mathbf{r}}-\vec{j}_{2} \mathbf{e}_{\mathbf{t}}+\vec{j}_{3} \mathbf{e}_{\mathbf{t r}}-\vec{j}_{4} i
$$

where $\rho_{\mathrm{s}}=4 \pi \rho_{\mathrm{s}}^{\prime}\left(\rho_{\mathrm{s}}^{\prime}\right.$ is the volume density of charge $)$ and $\vec{j}_{\mathrm{s}}=\frac{4 \pi}{c} \vec{j}_{\mathrm{s}}^{\prime}\left(\vec{j}_{\mathrm{s}}^{\prime}\right.$ is volume density of current). Multiplying the operators in the left part of equation (7) and separating the values with different space-time properties, we obtain the following wave equations for the components of potentials:

$$
\begin{aligned}
& \left(\partial^{2}-\Delta+m^{2}\right) a_{\mathrm{s}}=\rho_{\mathrm{s}}, \\
& \left(\partial^{2}-\Delta+m^{2}\right) \overrightarrow{A_{\mathrm{s}}}=\vec{j}_{\mathrm{s}},
\end{aligned}
$$

where $\triangle$ is the Laplace operator. Taking into account the definition (10) we choose the sedeon of field strength as

$$
\tilde{\mathbf{E}}=-\varepsilon_{1}+i \varepsilon_{2} \mathbf{e}_{\mathbf{t r}}+i \varepsilon_{3} \mathbf{e}_{\mathbf{t}}-i \varepsilon_{4} \mathbf{e}_{\mathbf{r}}+\vec{E}_{1} \mathbf{e}_{\mathbf{t r}}-i \vec{E}_{2}+\vec{E}_{3} \mathbf{e}_{\mathbf{r}}+\vec{E}_{4} \mathbf{e}_{\mathbf{t}}
$$


so we have the following definitions for scalar $\varepsilon_{\mathrm{s}}$ and vector $\vec{E}_{\mathrm{s}}$ field strengths:

$$
\begin{aligned}
& \varepsilon_{1}=\partial a_{1}+\left(\vec{\nabla} \cdot \overrightarrow{A_{1}}\right)+m a_{4}, \\
& \varepsilon_{2}=\partial a_{2}+\left(\vec{\nabla} \cdot \overrightarrow{A_{2}}\right)-m a_{3}, \\
& \varepsilon_{3}=\partial a_{3}+\left(\vec{\nabla} \cdot \overrightarrow{A_{3}}\right)+m a_{2}, \\
& \varepsilon_{4}=\partial a_{4}+\left(\vec{\nabla} \cdot \overrightarrow{A_{4}}\right)-m a_{1}, \\
& \vec{E}_{1}=-\partial \overrightarrow{A_{1}}-\vec{\nabla} a_{1}+i\left[\vec{\nabla} \times \overrightarrow{A_{2}}\right]+m \overrightarrow{A_{4}}, \\
& \vec{E}_{2}=-\partial \vec{A}_{2}-\vec{\nabla} a_{2}-i\left[\vec{\nabla} \times \overrightarrow{A_{1}}\right]-m \overrightarrow{A_{3}}, \\
& \vec{E}_{3}=-\partial \overrightarrow{A_{3}}-\vec{\nabla} a_{3}-i\left[\vec{\nabla} \times \overrightarrow{A_{4}}\right]+m \overrightarrow{A_{2}}, \\
& \vec{E}_{4}=-\partial \overrightarrow{A_{4}}-\vec{\nabla} a_{4}+i\left[\vec{\nabla} \times \overrightarrow{A_{3}}\right]-m \overrightarrow{A_{1}} .
\end{aligned}
$$

Thus, we have

$$
\begin{aligned}
& \widehat{\nabla}\left(i a_{1} \mathbf{e}_{\mathbf{t}}-i a_{2} \mathbf{e}_{\mathbf{r}}+a_{3}-i a_{4} \mathbf{e}_{\mathbf{t r}}+\vec{A}_{1} \mathbf{e}_{\mathbf{r}}+\vec{A}_{2} \mathbf{e}_{\mathbf{t}}-\vec{A}_{3} \mathbf{e}_{\mathbf{t r}}+i \vec{A}_{4}\right) \\
& =-\varepsilon_{1}+i \varepsilon_{2} \mathbf{e}_{\mathbf{t r}}+i \varepsilon_{3} \mathbf{e}_{\mathbf{t}}-i \varepsilon_{4} \mathbf{e}_{\mathbf{r}}+\vec{E}_{1} \mathbf{e}_{\mathbf{t r}}-i \vec{E}_{2}+\vec{E}_{3} \mathbf{e}_{\mathbf{r}}+\vec{E}_{4} \mathbf{e}_{\mathbf{t}},
\end{aligned}
$$

and the initial wave equation (7) takes the following form (see (11)) :

$$
\begin{aligned}
& \widehat{\nabla}\left(-\varepsilon_{1}+i \varepsilon_{2} \mathbf{e}_{\mathbf{t r}}+i \varepsilon_{3} \mathbf{e}_{\mathbf{t}}-i \varepsilon_{4} \mathbf{e}_{\mathbf{r}}+\vec{E}_{1} \mathbf{e}_{\mathbf{t r}}-i \vec{E}_{2}+\vec{E}_{3} \mathbf{e}_{\mathbf{r}}+\vec{E}_{4} \mathbf{e}_{\mathbf{t}}\right) \\
& =-i \rho_{1} \mathbf{e}_{\mathbf{t}}+i \rho_{2} \mathbf{e}_{\mathbf{r}}-\rho_{3}+i \rho_{4} \mathbf{e}_{\mathbf{t r}}-\vec{j}_{1} \mathbf{e}_{\mathbf{r}}-\vec{j}_{2} \mathbf{e}_{\mathbf{t}}+\vec{j}_{3} \mathbf{e}_{\mathbf{t r}}-\vec{j}_{4} i
\end{aligned}
$$

Producing the action of the operator on the left side of the equation (24) and separating the values with different space-time properties, we obtain a system of equations for the field strengths, similar to the system of Maxwell equations in electrodynamics:

$$
\begin{aligned}
& \partial \varepsilon_{1}+\left(\vec{\nabla} \cdot \vec{E}_{1}\right)-m \varepsilon_{4}=\rho_{1}, \\
& \partial \varepsilon_{2}+\left(\vec{\nabla} \cdot \vec{E}_{2}\right)+m \varepsilon_{3}=\rho_{2}, \\
& \partial \varepsilon_{3}+\left(\vec{\nabla} \cdot \vec{E}_{3}\right)-m \varepsilon_{2}=\rho_{3}, \\
& \partial \varepsilon_{4}+\left(\vec{\nabla} \cdot \vec{E}_{4}\right)+m \varepsilon_{1}=\rho_{4}, \\
& \partial \vec{E}_{1}+\vec{\nabla} \varepsilon_{1}+i\left[\vec{\nabla} \times \vec{E}_{2}\right]+m \vec{E}_{4}=-\vec{j}_{1}, \\
& \partial \vec{E}_{2}+\vec{\nabla} \varepsilon_{2}-i\left[\vec{\nabla} \times \vec{E}_{1}\right]-m \vec{E}_{3}=-\vec{j}_{2}, \\
& \partial \vec{E}_{3}+\vec{\nabla} \varepsilon_{3}-i\left[\vec{\nabla} \times \vec{E}_{4}\right]+m \vec{E}_{2}=-\vec{j}_{3}, \\
& \partial \vec{E}_{4}+\vec{\nabla} \varepsilon_{4}+i\left[\vec{\nabla} \times \vec{E}_{3}\right]-m \vec{E}_{1}=-\vec{j}_{4} .
\end{aligned}
$$

On the other hand, separating in equation (12) the terms with different space-time properties we 
get the following wave equation for the field strength components $\varepsilon_{\mathrm{s}}$ and $\vec{E}_{\mathrm{s}}$ :

$$
\begin{aligned}
& \left(\partial^{2}-\Delta+m^{2}\right) \varepsilon_{1}=-\partial \rho_{1}-\left(\vec{\nabla} \cdot \overrightarrow{j_{1}}\right)-m \rho_{4}, \\
& \left(\partial^{2}-\Delta+m^{2}\right) \varepsilon_{2}=-\partial \rho_{2}-\left(\vec{\nabla} \cdot \vec{j}_{2}\right)+m \rho_{3}, \\
& \left(\partial^{2}-\Delta+m^{2}\right) \varepsilon_{3}=-\partial \rho_{3}-\left(\vec{\nabla} \cdot \overrightarrow{j_{3}}\right)-m \rho_{2}, \\
& \left(\partial^{2}-\Delta+m^{2}\right) \varepsilon_{4}=-\partial \rho_{4}-\left(\vec{\nabla} \cdot \overrightarrow{j_{4}}\right)+m \rho_{1}, \\
& \left(\partial^{2}-\Delta+m^{2}\right) \vec{E}_{1}=\partial \vec{j}_{1}+\vec{\nabla} \rho_{1}-i\left[\vec{\nabla} \times \vec{j}_{2}\right]-m \vec{j}_{4}, \\
& \left(\partial^{2}-\Delta+m^{2}\right) \vec{E}_{2}=\partial \vec{j}_{2}+\vec{\nabla} \rho_{2}+i\left[\vec{\nabla} \times \vec{j}_{1}\right]+m \vec{j}_{3}, \\
& \left(\partial^{2}-\Delta+m^{2}\right) \vec{E}_{3}=\partial \vec{j}_{3}+\vec{\nabla} \rho_{3}+i\left[\vec{\nabla} \times \vec{j}_{4}\right]-m \vec{j}_{2}, \\
& \left(\partial^{2}-\Delta+m^{2}\right) \vec{E}_{4}=\partial \vec{j}_{4}+\vec{\nabla} \rho_{4}-i\left[\vec{\nabla} \times \vec{j}_{3}\right]+m \vec{j}_{1} .
\end{aligned}
$$

Let us consider the gauge invariance for the components of massive field. We take the arbitrary sedeons $\tilde{\mathbf{F}}$ and $\tilde{\mathbf{G}}$ in the following form:

$$
\begin{gathered}
\tilde{\mathbf{F}}=i f_{1} \mathbf{e}_{\mathbf{t}}-i f_{2} \mathbf{e}_{\mathbf{r}}+f_{3}-i f_{4} \mathbf{e}_{\mathbf{t r}}+\vec{F}_{1} \mathbf{e}_{\mathbf{r}}+\vec{F}_{2} \mathbf{e}_{\mathbf{t}}-\vec{F}_{3} \mathbf{e}_{\mathbf{t r}}+i \vec{F}_{4}, \\
\tilde{\mathbf{G}}=-g_{1}+i g_{2} \mathbf{e}_{\mathbf{t r}}+i g_{3} \mathbf{e}_{\mathbf{t}}-i g_{4} \mathbf{e}_{\mathbf{r}}-\vec{G}_{1} \mathbf{e}_{\mathbf{t r}}+i \vec{G}_{2}-\vec{G}_{3} \mathbf{e}_{\mathbf{r}}-\vec{G}_{4} \mathbf{e}_{\mathbf{t}} .
\end{gathered}
$$

Then the replacement (13) leads us to the following system of substitutions:

$$
\begin{aligned}
& a_{1} \Rightarrow a_{1}+f_{1}-\partial g_{1}+\left(\vec{\nabla} \cdot \vec{G}_{1}\right)+m g_{4}, \\
& a_{2} \Rightarrow a_{2}+f_{2}-\partial g_{2}+\left(\vec{\nabla} \cdot \vec{G}_{2}\right)-m g_{3}, \\
& a_{3} \Rightarrow a_{3}+f_{3}-\partial g_{3}+\left(\vec{\nabla} \cdot \vec{G}_{3}\right)+m g_{2}, \\
& a_{4} \Rightarrow a_{4}+f_{4}-\partial g_{4}+\left(\vec{\nabla} \cdot \vec{G}_{4}\right)-m g_{1}, \\
& \vec{A}_{1} \Rightarrow \vec{A}_{1}+\vec{F}_{1}-\partial \vec{G}_{1}+\vec{\nabla} g_{1}-i\left[\vec{\nabla} \times \vec{G}_{2}\right]-m \vec{G}_{4}, \\
& \vec{A}_{2} \Rightarrow \vec{A}_{2}+\vec{F}_{2}-\partial \vec{G}_{2}+\vec{\nabla} g_{2}+i\left[\vec{\nabla} \times \vec{G}_{1}\right]+m \vec{G}_{3}, \\
& \vec{A}_{3} \Rightarrow \vec{A}_{3}+\vec{F}_{3}-\partial \vec{G}_{3}+\vec{\nabla} g_{3}+i\left[\vec{\nabla} \times \vec{G}_{4}\right]-m \vec{G}_{2}, \\
& \vec{A}_{4} \Rightarrow \vec{A}_{4}+\vec{F}_{4}-\partial \vec{G}_{4}+\vec{\nabla} g_{4}-i\left[\vec{\nabla} \times \vec{G}_{3}\right]+m \vec{G}_{1} .
\end{aligned}
$$

It is easy to check that these replacements do not change the field strengths definitions (22) and corresponding equations (25) and (26). Analogous substitutions for the field strengths have the 
following form:

$$
\begin{aligned}
\varepsilon_{1} & \Rightarrow \varepsilon_{1}-f_{3}+\partial g_{3}-\left(\vec{\nabla} \cdot \vec{G}_{3}\right)-m g_{2}, \\
\varepsilon_{2} & \Rightarrow \varepsilon_{2}-f_{4}+\partial g_{4}-\left(\vec{\nabla} \cdot \vec{G}_{4}\right)+m g_{1}, \\
\varepsilon_{3} & \Rightarrow \varepsilon_{3}+f_{1}-\partial g_{1}+\left(\vec{\nabla} \cdot \vec{G}_{1}\right)+m g_{4}, \\
\varepsilon_{4} & \Rightarrow \varepsilon_{4}+f_{2}-\partial g_{2}+\left(\vec{\nabla} \cdot \vec{G}_{2}\right)-m g_{3}, \\
\vec{E}_{1} & \Rightarrow \vec{E}_{1}+\vec{F}_{3}-\partial \vec{G}_{3}+\vec{\nabla} g_{3}+i\left[\vec{\nabla} \times \vec{G}_{4}\right]-m \vec{G}_{2}, \\
\vec{E}_{2} & \Rightarrow \vec{E}_{2}+\vec{F}_{4}-\partial \vec{G}_{4}+\vec{\nabla} g_{4}-i\left[\vec{\nabla} \times \vec{G}_{3}\right]+m \vec{G}_{1}, \\
\vec{E}_{3} & \Rightarrow \vec{E}_{3}-\vec{F}_{1}+\partial \vec{G}_{1}-\vec{\nabla} g_{1}+i\left[\vec{\nabla} \times \vec{G}_{2}\right]+m \vec{G}_{4}, \\
\vec{E}_{4} & \Rightarrow \vec{E}_{4}-\vec{F}_{2}+\partial \vec{G}_{2}-\vec{\nabla} g_{2}-i\left[\vec{\nabla} \times \vec{G}_{1}\right]-m \vec{G}_{3} .
\end{aligned}
$$

These substitutions do not change the equations (25).

Let us discuss the Proca-Maxwell equations for massive fields. If we consider the fields produced by a one type of sources $\rho_{1}$ and $\vec{j}_{1}$ and take very strict condition, that the massive field is described only by $a_{1}$ and $\overrightarrow{A_{1}}$ potentials:

$$
\tilde{\mathbf{W}}=i a_{1} \mathbf{e}_{\mathbf{t}}+\vec{A}_{1} \mathbf{e}_{\mathbf{r}},
$$

then we have only the following nonzero field's strengths (see (22)):

$$
\begin{aligned}
& \varepsilon_{1}=\partial a_{1}+\left(\vec{\nabla} \cdot \overrightarrow{A_{1}}\right), \\
& \varepsilon_{4}=-m a_{1}, \\
& \vec{E}_{1}=-\partial \overrightarrow{A_{1}}-\vec{\nabla} a_{1}, \\
& \vec{E}_{2}=-i\left[\vec{\nabla} \times \overrightarrow{A_{1}}\right], \\
& \vec{E}_{4}=-m \overrightarrow{A_{1}} .
\end{aligned}
$$

Moreover, often they assume the charge conservation

$$
\partial \rho_{1}+\left(\nabla \cdot \vec{j}_{1}\right)=0,
$$

and choose the scalar field $\varepsilon_{1}$ equal to zero (see the first equation in (26). It is equivalent to the following gauge condition:

$$
\partial a_{1}+\left(\vec{\nabla} \cdot \overrightarrow{A_{1}}\right)=0,
$$

which coincides with Lorentz gauge in electrodynamics. Then the wave equation (24) can be reduce to

$$
\begin{aligned}
& \left(i \mathbf{e}_{\mathbf{t}} \partial-\mathbf{e}_{\mathbf{r}} \vec{\nabla}-i \mathbf{e}_{\mathbf{t r}} m\right)\left(-i \varepsilon_{4} \mathbf{e}_{\mathbf{r}}+\vec{E}_{1} \mathbf{e}_{\mathbf{t r}}-i \vec{E}_{2}+\vec{E}_{4} \mathbf{e}_{\mathbf{t}}\right) \\
& =-i \rho_{1} \mathbf{e}_{\mathbf{t}}-\vec{j}_{1} \mathbf{e}_{\mathbf{r}},
\end{aligned}
$$


and the system (25) takes the following form:

$$
\begin{aligned}
& \left(\vec{\nabla} \cdot \vec{E}_{1}\right)-m \varepsilon_{4}=\rho_{1}, \\
& \left(\vec{\nabla} \cdot \vec{E}_{2}\right)=0, \\
& \partial \varepsilon_{4}+\left(\vec{\nabla} \cdot \vec{E}_{4}\right)=0, \\
& \partial \vec{E}_{1}+i\left[\vec{\nabla} \times \vec{E}_{2}\right]+m \vec{E}_{4}=-\vec{j}_{1}, \\
& \partial \vec{E}_{2}-i\left[\vec{\nabla} \times \vec{E}_{1}\right]=0, \\
& i\left[\vec{\nabla} \times \vec{E}_{4}\right]-m \vec{E}_{2}=0, \\
& \partial \vec{E}_{4}+\vec{\nabla}_{4}-m \vec{E}_{1}=0 .
\end{aligned}
$$

The system (36) is the analog of Proca-Maxwell equations with considerably reduced symmetry and broken gauge invariance.

\section{Second-order equation for massless field}

Let us consider the symmetric equation for massless electromagnetic field taking into account the hypothetical magnetic charge (magnetic monopole) and corresponding magnetic current $[19,20]$. In massless case the wave equation (7) takes the following form:

$$
\left(i \mathbf{e}_{\mathbf{t}} \partial-\mathbf{e}_{\mathbf{r}} \vec{\nabla}\right)\left(i \mathbf{e}_{\mathbf{t}} \partial-\mathbf{e}_{\mathbf{r}} \vec{\nabla}\right) \tilde{\mathbf{W}}=\tilde{\mathbf{J}}
$$

When the mass of the field quantum equal to zero, the equations (25) are disengaged and the field can be described by the eight-component potential

$$
\tilde{\mathbf{W}}=i a_{1} \mathbf{e}_{\mathbf{t}}-i a_{2} \mathbf{e}_{\mathbf{r}}+\vec{A}_{1} \mathbf{e}_{\mathbf{r}}+\vec{A}_{2} \mathbf{e}_{\mathbf{t}}
$$

and eight-component source

$$
\tilde{\mathbf{J}}=-i \rho_{1} \mathbf{e}_{\mathbf{t}}+i \rho_{2} \mathbf{e}_{\mathbf{r}}-\vec{j}_{1} \mathbf{e}_{\mathbf{r}}-\vec{j}_{2} \mathbf{e}_{\mathbf{t}}
$$

Here $a_{1}$ and $\overrightarrow{A_{1}}$ are scalar and vector electric potentials; $a_{2}$ and $\vec{A}_{2}$ are scalar and vector magnetic potentials; $\rho_{1}$ and $\vec{j}_{1}$ are the volume density of electric charge and current; $\rho_{2}$ and $\vec{j}_{2}$ are the volume density of magnetic charge and current. We can introduce the scalar and vector field strengths according the following definitions:

$$
\begin{aligned}
& \varepsilon_{1}=\partial a_{1}+\left(\vec{\nabla} \cdot \overrightarrow{A_{1}}\right), \\
& \varepsilon_{2}=\partial a_{2}+\left(\vec{\nabla} \cdot \overrightarrow{A_{2}}\right), \\
& \vec{E}_{1}=-\partial \overrightarrow{A_{1}}-\vec{\nabla} a_{1}+i\left[\vec{\nabla} \times \overrightarrow{A_{2}}\right], \\
& \vec{E}_{2}=-\partial \overrightarrow{A_{2}}-\vec{\nabla} a_{2}-i\left[\vec{\nabla} \times \overrightarrow{A_{1}}\right] .
\end{aligned}
$$

Taking into account (40) we get

$$
\begin{aligned}
& \left(i \mathbf{e}_{\mathbf{t}} \partial-\mathbf{e}_{\mathbf{r}} \vec{\nabla}\right)\left(i a_{1} \mathbf{e}_{\mathbf{t}}-i a_{2} \mathbf{e}_{\mathbf{r}}+\vec{A}_{1} \mathbf{e}_{\mathbf{r}}+\vec{A}_{2} \mathbf{e}_{\mathbf{t}}\right) \\
& =-\varepsilon_{1}+i \varepsilon_{2} \mathbf{e}_{\mathbf{t r}}+\vec{E}_{1} \mathbf{e}_{\mathbf{t r}}-i \vec{E}_{2},
\end{aligned}
$$

and wave equation $(37)$ can be rewritten as

$$
\begin{aligned}
& \left(i \mathbf{e}_{\mathbf{t}} \partial-\mathbf{e}_{\mathbf{r}} \vec{\nabla}\right)\left(-\varepsilon_{1}+i \varepsilon_{2} \mathbf{e}_{\mathbf{t r}}+\vec{E}_{1} \mathbf{e}_{\mathbf{t r}}-i \vec{E}_{2}\right) \\
& =-i \rho_{1} \mathbf{e}_{\mathbf{t}}+i \rho_{2} \mathbf{e}_{\mathbf{r}}-\vec{j}_{1} \mathbf{e}_{\mathbf{r}}-\vec{j}_{2} \mathbf{e}_{\mathbf{t}} .
\end{aligned}
$$


Producing the action of the operator on the left side of equation (42) and separating the terms with different space-time properties, we obtain the symmetric system of the equations for the field strengths

$$
\begin{aligned}
& \partial \varepsilon_{1}+\left(\vec{\nabla} \cdot \vec{E}_{1}\right)=\rho_{1}, \\
& \partial \varepsilon_{2}+\left(\vec{\nabla} \cdot \vec{E}_{2}\right)=\rho_{2}, \\
& \partial \vec{E}_{1}+\vec{\nabla} \varepsilon_{1}+i\left[\vec{\nabla} \times \vec{E}_{2}\right]=-\vec{j}_{1}, \\
& \partial \vec{E}_{2}+\vec{\nabla} \varepsilon_{2}-i\left[\vec{\nabla} \times \vec{E}_{1}\right]=-\vec{j}_{2} .
\end{aligned}
$$

Corresponding wave equations for the field strengths have the following form:

$$
\begin{aligned}
& \left(\partial^{2}-\Delta\right) \varepsilon_{1}=-\partial \rho_{1}-\left(\vec{\nabla} \cdot \vec{j}_{1}\right), \\
& \left(\partial^{2}-\Delta\right) \varepsilon_{2}=-\partial \rho_{2}-\left(\vec{\nabla} \cdot \vec{j}_{2}\right), \\
& \left(\partial^{2}-\Delta\right) \vec{E}_{1}=\vec{\nabla} \rho_{1}+\partial \vec{j}_{1}-i\left[\vec{\nabla} \times \vec{j}_{2}\right], \\
& \left(\partial^{2}-\Delta\right) \vec{E}_{2}=\vec{\nabla} \rho_{2}+\partial \vec{j}_{2}+i\left[\vec{\nabla} \times \vec{j}_{1}\right] .
\end{aligned}
$$

The definitions of massless field strengths (40) and the equations (44) are not changed under the following replacements:

$$
\begin{aligned}
& a_{1} \Rightarrow a_{1}+f_{1}-\partial g_{1}+\left(\vec{\nabla} \cdot \vec{G}_{1}\right), \\
& a_{2} \Rightarrow a_{2}+f_{2}-\partial g_{2}+\left(\vec{\nabla} \cdot \vec{G}_{2}\right), \\
& \vec{A}_{1} \Rightarrow \vec{A}_{1}+\vec{F}_{1}-\partial \vec{G}_{1}+\vec{\nabla} g_{1}-i\left[\vec{\nabla} \times \vec{G}_{2}\right], \\
& \vec{A}_{2} \Rightarrow \vec{A}_{2}+\vec{F}_{2}-\partial \vec{G}_{2}+\vec{\nabla} g_{2}+i\left[\vec{\nabla} \times \vec{G}_{1}\right] .
\end{aligned}
$$

The gauge conditions (45) include the gradient invariance relations well known in classical electrodynamics [21] as a particular case, when values $f_{1}, f_{2}, \vec{F}_{1}, \vec{F}_{2}, \vec{G}_{1}, \vec{G}_{2}$ are equal to zero. Analogously the equation (43) is not changed under following substitutions for the field strengths :

$$
\begin{aligned}
& \varepsilon_{1} \Rightarrow \varepsilon_{1}-f_{3}+\partial g_{3}-\left(\vec{\nabla} \cdot \vec{G}_{3}\right), \\
& \varepsilon_{2} \Rightarrow \varepsilon_{2}-f_{4}+\partial g_{4}-\left(\vec{\nabla} \cdot \vec{G}_{4}\right), \\
& \vec{E}_{1} \Rightarrow \vec{E}_{1}+\vec{F}_{3}-\partial \vec{G}_{3}+\vec{\nabla} g_{3}+i\left[\vec{\nabla} \times \vec{G}_{4}\right], \\
& \vec{E}_{2} \Rightarrow \vec{E}_{2}+\vec{F}_{4}-\partial \vec{G}_{4}+\vec{\nabla} g_{4}-i\left[\vec{\nabla} \times \vec{G}_{3}\right] .
\end{aligned}
$$

The system of equations (43) corresponds to the usual system of Maxwell equations. Let us show it. If we take the scalar fields $\varepsilon_{1}$ and $\varepsilon_{2}$ equal to zero then the potentials satisfy the Lorentz gauge conditions for electric and magnetic potentials:

$$
\begin{aligned}
& \partial a_{1}+\left(\vec{\nabla} \cdot \overrightarrow{A_{1}}\right)=0, \\
& \partial a_{2}+\left(\vec{\nabla} \cdot \vec{A}_{2}\right)=0,
\end{aligned}
$$

and as it follows from (44) we have charge conservation

$$
\begin{aligned}
& \partial \rho_{1}+\left(\vec{\nabla} \cdot \vec{j}_{1}\right)=0, \\
& \partial \rho_{2}+\left(\vec{\nabla} \cdot \vec{j}_{2}\right)=0 .
\end{aligned}
$$


Then we obtain the following system:

$$
\begin{aligned}
& \left(\vec{\nabla} \cdot \vec{E}_{1}\right)=\rho_{1}, \\
& \left(\vec{\nabla} \cdot \vec{E}_{2}\right)=\rho_{2}, \\
& \partial \vec{E}_{1}+i\left[\vec{\nabla} \times \vec{E}_{2}\right]=-\vec{j}_{1}, \\
& \partial \vec{E}_{2}-i\left[\vec{\nabla} \times \vec{E}_{1}\right]=-\vec{j}_{2} .
\end{aligned}
$$

Here $\vec{E}_{1}$ is the electric field strength, $\vec{E}_{2}$ is the magnetic field strength. Taking into account the experimental fact that in our part of the universe there are no magnetic charges and currents $\left(\rho_{2}=0, \vec{j}_{2}=0\right)$, we obtain the system of equations

$$
\begin{aligned}
& \left(\vec{\nabla} \cdot \vec{E}_{1}\right)=\rho_{1}, \\
& \left(\vec{\nabla} \cdot \vec{E}_{2}\right)=0, \\
& \partial \vec{E}_{1}+i\left[\vec{\nabla} \times \vec{E}_{2}\right]=-\vec{j}_{1}, \\
& \partial \vec{E}_{2}-i\left[\vec{\nabla} \times \vec{E}_{1}\right]=0,
\end{aligned}
$$

which coincides with the conventional system of Maxwell's equations.

\section{First-order wave equation of massive field}

Let us consider a massive field, which is described by the sedeonic first-order wave equation [18]:

$$
\bar{\nabla} \tilde{\mathbf{W}}=\tilde{\mathbf{I}} .
$$

Here $\tilde{\mathbf{I}}$ is the phenomenological field source. The field source can be chosen in the following sedeonic form:

$$
\tilde{\mathbf{I}}=-\beta_{1}+i \beta_{2} \mathbf{e}_{\mathbf{t r}}+i \beta_{3} \mathbf{e}_{\mathbf{t}}-i \beta_{4} \mathbf{e}_{\mathbf{r}}+\overrightarrow{l_{1}} \mathbf{e}_{\mathbf{t r}}-i \overrightarrow{l_{2}}+\overrightarrow{l_{3}} \mathbf{e}_{\mathbf{r}}+\vec{l}_{4} \mathbf{e}_{\mathbf{t}}
$$

where $\beta_{\mathrm{s}}=4 \pi \beta_{\mathrm{s}}^{\prime}$ ( $\beta_{\mathrm{s}}^{\prime}$ are the volume density of charges) and $\vec{l}_{\mathrm{s}}=\frac{4 \pi}{c} \vec{l}_{\mathrm{s}}^{\prime}\left(\vec{l}_{\mathrm{s}}^{\prime}\right.$ are the corresponding volume density of currents). Taking the potential $\tilde{\mathbf{W}}$ in the form of (18) we can rewrite the equation (51) in the following expanded form

$$
\begin{aligned}
& \stackrel{\nabla}{\nabla}\left(i a_{1} \mathbf{e}_{\mathbf{t}}-i a_{2} \mathbf{e}_{\mathbf{r}}+a_{3}-i a_{4} \mathbf{e}_{\mathbf{t r}}+\vec{A}_{1} \mathbf{e}_{\mathbf{r}}+\vec{A}_{2} \mathbf{e}_{\mathbf{t}}-\vec{A}_{3} \mathbf{e}_{\mathbf{t r}}+i \vec{A}_{4}\right) \\
& =-\beta_{1}+i \beta_{2} \mathbf{e}_{\mathbf{t r}}+i \beta_{3} \mathbf{e}_{\mathbf{t}}-i \beta_{4} \mathbf{e}_{\mathbf{r}}+\vec{l}_{1} \mathbf{e}_{\mathbf{t r}}-i \vec{l}_{2}+\overrightarrow{f_{3}} \mathbf{e}_{\mathbf{r}}+\vec{l}_{4} \mathbf{e}_{\mathbf{t}} .
\end{aligned}
$$

This sedeonic equation is equivalent to the following system:

$$
\begin{aligned}
& \partial a_{1}+\left(\vec{\nabla} \cdot \overrightarrow{A_{1}}\right)+m a_{4}=\beta_{1}, \\
& \partial a_{2}+\left(\vec{\nabla} \cdot \overrightarrow{A_{2}}\right)-m a_{3}=\beta_{2}, \\
& \partial a_{3}+\left(\vec{\nabla} \cdot \overrightarrow{A_{3}}\right)+m a_{2}=\beta_{3}, \\
& \partial a_{4}+\left(\vec{\nabla} \cdot \overrightarrow{A_{4}}\right)-m a_{1}=\beta_{4}, \\
& \partial \overrightarrow{A_{1}}+\vec{\nabla} a_{1}-i\left[\vec{\nabla} \times \overrightarrow{A_{2}}\right]-m \overrightarrow{A_{4}}=-\overrightarrow{l_{1}}, \\
& \partial \overrightarrow{A_{2}}+\vec{\nabla} a_{2}+i\left[\vec{\nabla} \times \overrightarrow{A_{1}}\right]+m \vec{A}_{3}=-\overrightarrow{l_{2}}, \\
& \partial \overrightarrow{A_{3}}+\vec{\nabla} a_{3}+i\left[\vec{\nabla} \times \overrightarrow{A_{4}}\right]-m \overrightarrow{A_{2}}=-\vec{l}_{3}, \\
& \partial \overrightarrow{A_{4}}+\vec{\nabla} a_{4}-i\left[\vec{\nabla} \times \overrightarrow{A_{3}}\right]+m \overrightarrow{A_{1}}=-\vec{l}_{4} .
\end{aligned}
$$


Applying the operator $\widehat{\nabla}$ to the equation (51) we obtain the following second-order wave equation:

$$
\widehat{\nabla} \widehat{\nabla} \tilde{\mathbf{W}}=\widehat{\nabla} \tilde{\mathbf{I}}
$$

which is equivalent to the following system:

$$
\begin{aligned}
& \left(\partial^{2}-\Delta+m^{2}\right) a_{1}=\partial \beta_{1}+\left(\vec{\nabla} \cdot \vec{l}_{1}\right)-m d_{4}, \\
& \left(\partial^{2}-\Delta+m^{2}\right) a_{2}=\partial \beta_{2}+\left(\vec{\nabla} \cdot \vec{l}_{2}\right)+m d_{3}, \\
& \left(\partial^{2}-\Delta+m^{2}\right) a_{3}=\partial \beta_{3}+\left(\vec{\nabla} \cdot \vec{l}_{3}\right)-m d_{2}, \\
& \left(\partial^{2}-\Delta+m^{2}\right) a_{4}=\partial \beta_{4}+\left(\vec{\nabla} \cdot \vec{l}_{4}\right)+m d_{1}, \\
& \left(\partial^{2}-\Delta+m^{2}\right) \vec{A}_{1}=-\partial \overrightarrow{l_{1}}-\vec{\nabla} \beta_{1}-i\left[\vec{\nabla} \times \vec{l}_{2}\right]-m \vec{l}_{4}, \\
& \left(\partial^{2}-\Delta+m^{2}\right) \vec{A}_{2}=-\partial \overrightarrow{l_{2}}-\vec{\nabla} \beta_{2}+i\left[\vec{\nabla} \times \overrightarrow{l_{1}}\right]+m \vec{l}_{3}, \\
& \left(\partial^{2}-\Delta+m^{2}\right) \vec{A}_{3}=-\partial \overrightarrow{l_{3}}-\vec{\nabla} \beta_{3}+i\left[\vec{\nabla} \times \vec{l}_{4}\right]-m \vec{l}_{2}, \\
& \left(\partial^{2}-\Delta+m^{2}\right) \vec{A}_{4}=-\partial \vec{l}_{4}-\vec{\nabla} \beta_{4}-i\left[\vec{\nabla} \times \vec{l}_{3}\right]+m \vec{l}_{1},
\end{aligned}
$$

It can be seen that as before the equation (51) is invariant with respect to the following potential replacement:

$$
\tilde{\mathbf{W}} \Rightarrow \tilde{\mathbf{W}}+\tilde{\mathbf{F}}+\hat{\nabla} \tilde{\mathbf{G}} .
$$

In addition, the equation (55) is invariant for the following substitution for the source:

$$
\tilde{\mathbf{I}} \Rightarrow \tilde{\mathbf{I}}+\tilde{\mathbf{F}}+\widehat{\nabla} \tilde{\mathbf{G}} .
$$

The generalization of all considerations for the case of massless field described by first-order wave equation is evident.

\section{Concluding remarks}

Thus it was shown that the approach based on sedeonic potentials and sedeonic space-time operators allows us to formulate the symmetric scalar-vector equations describing massive and massless fields. The gradient gauge invariance of the sedeonic equations is the specific property of the operator $\left(i \mathbf{e}_{\mathbf{t}} \partial-\mathbf{e}_{\mathbf{r}} \vec{\nabla}-i \mathbf{e}_{\mathbf{t r}} m\right)$ and can be generalized to a wider class of scalar-vector substitutions. On the example of Proca-Maxwell equations we have shown that the reduction of the number of potential components leads us to the breaking of symmetry and to the gauge invariance violation. On the other hand, we have shown that the symmetric definition of the field strengths in electrodynamics using both electric and magnetic potentials enables the generalization of gradient invariance on the considerably wider class of gauge functions.

\section{Acknowledgements}

The authors are very thankful to G.V. Mironova for kind assistance and moral support.

\section{References}

[1] S.L.Adler Quaternionic quantum mechanics and quantum fields, New York: Oxford University Press, 1995. 
[2] S. Ulrych - The Poincare mass operator in terms of a hyperbolic algebra, Physics Letters B, 612(1-2), 89 (2005).

[3] A.J. Davies - Quaternionic Dirac equation, Physical Review D, 41(8), 2628 (1990).

[4] S. De Leo and P. Rotelli - Quaternion scalar field, Physical Review D, 45(2), 575 (1992).

[5] S. Demir and M. Tanisli - A compact biquaternionic formulation of massive field equations in gravi-electromagnetism, European Physical Journal - Plus, 126(11), 115 (2011).

[6] J. Köplinger - Dirac equation on hyperbolic octonions, Applied Mathematics and Computation, 182, 443 (2006).

[7] C. Cafaro and S.A. Ali - The spacetime algebra approach to massive classical electrodynamics with magnetic monopoles, Advances in Applied Clifford Algebras, 17, 23 (2006).

[8] J. D. Jackson and L. B. Okun - Historical roots of gauge invariance, Reviews of Modern Physics, 73(3), 663 (2001).

[9] L.-Ch. Tu, J. Luo, and G. T. Gillies - The mass of the photon, Reports on Progress in Physisc, 68, 77 (2005).

[10] N. Candemir, M. Tanisli, K. Ozdas, and S. Demir - Hyperbolic octonionic Proca-Maxwell equations, Zeitschrift für Naturforschung A, 63a, 15 (2008).

[11] L.E. Dickson - On quaternions and their generalization and the history of the eight square theorem, Annals of Mathematics (SecondSeries), 20(3), 155 (1919).

[12] K. Carmody - Circular and hyperbolic quaternions, octonions, and sedenions, Applied Mathematics and Computation, 28, 47 (1988).

[13] K. Carmody - Circular and hyperbolic quaternions, octonions, and sedenions - further results, Applied Mathematics and Computation, 84, 27 (1997).

[14] K. Imaeda, M. Imaeda - Sedenions: algebra and analysis, Applied Mathematics and Computations, 115, 77 (2000).

[15] S. Demir and M. Tanisli - Sedenionic formulation for generalized fields of dyons, International Journal of Theoretical Physics, 51(4), 1239 (2012).

[16] W.P. Joyce - Dirac theory in spacetime algebra: I. The generalized bivector Dirac equation, Journal of Physics A: Mathematical and General, 34, 1991 (2001).

[17] V.L. Mironov and S.V. Mironov - Reformulation of relativistic quantum mechanics equations with non-commutative sedeons, Applied Mathematics, 4(10C), 53 (2013).

[18] S.V. Mironov and V.L. Mironov - Sedeonic equations of massive fields, International Journal of Theoretical Physics, 54(1), 153 (2015).

[19] P.A.M. Dirac Quantised singularities in the electromagnetic field, Proceedings of Royal Society at London. Ser. A., 133, 60-72 (1931). doi:10.1098/rspa.1931.0129 29.

[20] P.A.M. Dirac The theory of magnetic poles, Physical Review, 74, 817 (1948). 30. J.Schwinger

[21] L.D. Landau and E.M. Lifshits - Classical Theory of Fields (New York: Pergamon Press, 1975). 